\title{
Diversidad larval de mosquitos (Diptera: Culicidae) en contenedores artificiales procedentes de una comunidad urbana de San José, Costa Rica
}

\author{
OLGER CALDERÓN-ARGUEDAS*, ADRIANA TROYO* y MAYRA E. SOLANO*
}

\section{LARVAL DIVERSITY OF MOSQUITOES (DIPTERA: CULICIDAE) IN ARTIFICIAL CONTAINERS FROM AN URBAN COMMUNITY OF SAN JOSÉ, COSTA RICA}

A larval survey of mosquitoes was performed in artificial containers from indoor and outdoor enviroments in the neighborhood "La Carpio", San José, Costa Rica. "La Carpio" is a community that suffered a dengue outbreak during the last months of the rainy season in 2002. During 2003, 582 and 369 houses were visited in the dry and rainy season, respectively, and the potencial mosquito breeding sites were searched for larvae. Of 1160 water containers identified, 152 (13.0\%) were positive for at least one species of mosquito larvae and two mosquito species were found in $9(0.78 \%)$ of the containers. In order to estimate the mosquito diversity, an infestation index by species (Ii) was calculated for each kind of container. Aedes aegypti, Culex quinquefasciatus, Cx. restuans, Cx. coronator, and Limatus durhamii were identified. Ae. aegypti was the most common species, and it showed the highest infestation index for all the containers $(p<0.05)$. In addition to Ae. aegypti, the presence of other species of Culicidae could mask the success of campaigns for prevention of dengue. This is also an important factor to consider in order to prevent the ocurrence of emerging vector borne diseases such as West Nile Virus Encephalitis. Virus.

Key words: Aedes aegypti, Culex quinquefasciatus, dengue, mosquitoes, Culicidae, West Nile

\section{INTRODUCCIÓN}

La ciudadela "La Carpio", constituyó el escenario del primer brote epidémico de dengue en el Gran Área Metropolitana de San José, Costa Rica. Este tuvo lugar entre los meses de Agosto y Noviembre del 2002, meses que coinciden con la culminación de la estación lluviosa en el centro de Costa Rica y en los cuales se presenta la mayor incidencia de la virosis.
Las acciones de control desplegadas a raíz de dicho evento comprendieron el tratamiento focal y perifocal con insecticidas, la eliminación de criaderos, así como la información y educación de la población en lo referente al tema.

Como se ha determinado en otros estudios, cuando se implementa una campaña para la eliminación de un vector como Ae. aegypti, es esperable que otras especies de culícidos que utilizan un ámbito más amplio de depósitos de agua

\footnotetext{
* Centro de Investigación en Enfermedades Tropicales (CIET), Departamento de Parasitología, Facultad de Microbiología, Universidad de Costa Rica.E-mail: olgerc@ cariari.ucr.ac.cr
} 
(naturales y artificiales) para su multiplicación, sucedan a la primera, ocupando su hábitat ${ }^{1-3}$. Esto tiene lugar ya que, ante una disminución en las poblaciones larvales de Ae. aegypti, quedan disponibles gran cantidad de ambientes que resultan óptimos para ser recolonizados. Por lo tanto la presencia de estas especies de mosquitos pueden enmascarar las acciones de prevención y control ante la infestación por Ae. aegypti. Además, constituyen una variable epidemiológica a considerar ante la potencial ocurrencia de otras enfermedades de transmisión vectorial. En este sentido, es importante destacar la presencia continental del virus del Nilo Occidental (WNV), el cual es transmitido por estos dípteros y durante el último quinquenio ha presentado una expansión muy importante a partir de la costa este de América del Norte 4 .

En el presente estudio se identificaron las especies de culícidos cuyas larvas se suelen encontrar en depósitos de agua artificiales en la comunidad "La Carpio".

\section{MATERIAL Y MÉTODOS}

Como parte de un estudio sobre la caracterización de la infestación por Ae. aegypti en la comunidad "La Carpio", durante las estaciones seca y lluviosa del 2003 se realizó una encuesta entomológica para evaluar la presencia y diversidad de larvas de mosquitos (Diptera: Culicidae) en contenedores de agua artificiales pertenecientes a viviendas de esta localidad.

"La Carpio" es un caserío ubicado $7 \mathrm{Km}$ al oeste del centro urbano de San José, Costa Rica; cuenta con 2.705 viviendas y una población de aproximadamente 30.000 habitantes. El muestreo realizado fue de tipo sistemático y el tamaño de la muestra fue establecido de acuerdo a los criterios definidos por la Organización Panamericana de la Salud (OPS) para una confiabilidad del $95 \%$.

582 viviendas fueron visitadas en la estación seca y 369 en la estación lluviosa. Durante cada visita se identificaron los contenedores domiciliares y peridomiciliares que contenían agua acumulada de forma no transitoria y se colectó una muestra de las larvas de mosquitos presentes en cada contenedor positivo.

El material entomológico colectado se colocó en recipientes de cristal de $64 \times 7 \mathrm{~mm}$ con alcohol al $70 \%$ para su fijación y transporte al laboratorio.
Las larvas fueron aclaradas en lactofenol y montadas en medio Hoyer para su observación microscópica. La identificación de las mismas se realizó usando claves dicotómicas especializadas ${ }^{6-9}$.

Con el fin de estimar la diversidad, se calculó un índice de infestación por especie para cada tipo de criadero (Ii), de acuerdo a criterios establecidos ${ }^{2}$. Los Ii promedio para cada especie de mosquitos se compararon mediante pruebas de $t$ student, utilizando un coeficiente de confiabilidad del $95 \%$

\section{RESULTADOS}

La encuesta entomológica permitió identificar 1.160 potenciales criaderos artificiales para mosquitos urbanos, ubicados en las siguientes categorías: estañones, baldes, macetas, floreros, llantas, pilas, bebederos de animales, latas y objetos misceláneos. 152 de los contenedores $(13,0 \%)$ contenían al menos una especie de larvas de mosquito y 9 del total $(0,78 \%)$ mostraron dos especies coexistiendo en el mismo contenedor (Figuras 1 y 2).

Las especies identificadas fueron Ae. aegypti, Culex quinquefasciatus, $C x$. restuans, $C x$. coronator y Limatus durhamii. Ae. aegypti y $C x$. quinquefasciatus se observaron tanto en la estación seca como en la lluviosa, mientras que las especies restantes sólo fueron evidenciadas en la estación lluviosa (Tablas 1 y 2). Ae. aegypti, seguido de $C x$. quinquefasciatus, fue la especie que se encontró con mayor frecuencia en los

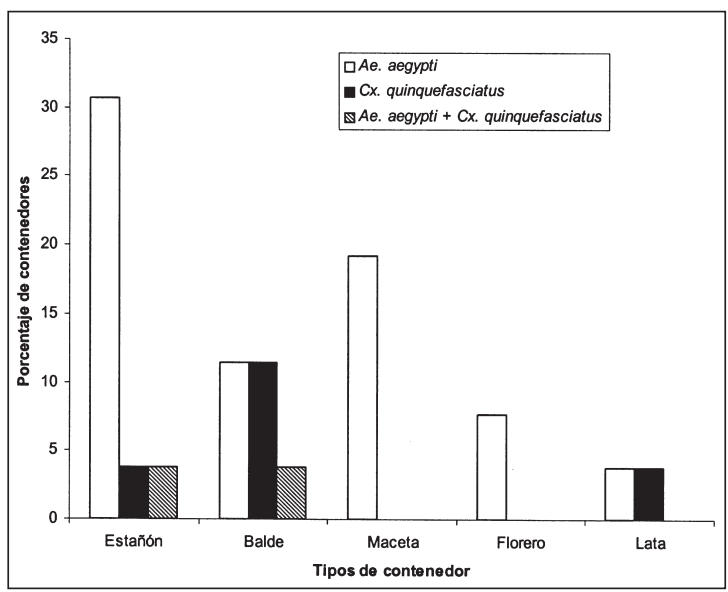

Figura 1. Porcentaje de contenedores positivos por larvas de culícidos durante la estación seca de 2003 en el caserío "La Carpio". 


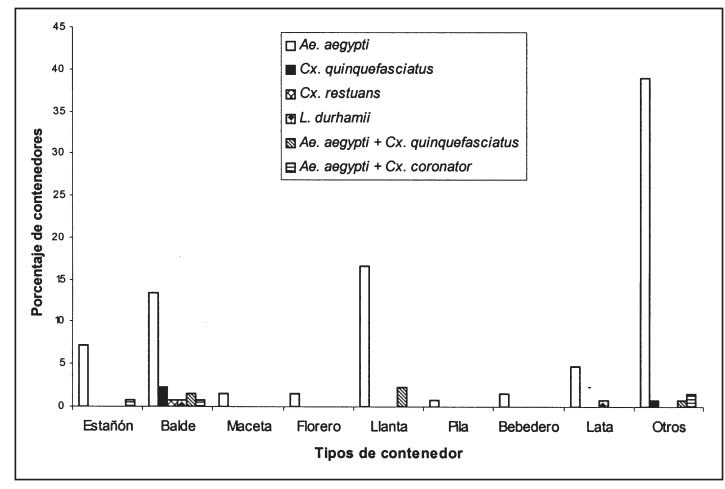

Figura 2. Porcentaje de contenedores positivos por larvas de culícidos durante la estación lluviosa de 2003.

contenedores evaluados por lo que sus índices de infestación por especie (Ii) fueron los más elevados $(\mathrm{p}<0,05)$ (Tablas 1 y 2$)$.

\section{DISCUSIÓN}

La presencia de Ae. aegypti como mosquito dominante en una población urbana ha sido uno de los hallazgos más típicos en los diferentes países de la región neotropical ${ }^{1,2}$. Ae. aegypti tiene un comportamiento eminentemente sinantrópico, con hábitat multiplicativos bastante definidos. En el presente estudio dicha especie se presentó como la más frecuente y mostró los índices de diversidad por especie más elevados para cada categoría de criadero (Tablas 1 y 2).

Al igual que lo sucedido en otras ciudades que se han visto aquejadas por epidemias de dengue, sería esperable que las medidas preventivas relacionadas con la eliminación y tratamiento de criaderos de Ae. aegypti se tradujeran en una disminución en su frecuencia y diversidad ${ }^{2}$. Sin embargo, en el presente estudio, Ae. aegypti se presentó como la especie dominante en la comunidad, aún después de las campañas de prevención y control contra este mosquito llevadas a cabo durante el año 2002. Lo anterior podría estar relacionado con diversos aspectos que pueden abarcar desde los factores inherentes a los vectores, tales como la ocurrencia de poblaciones residuales, resistencia a insecticidas o recolonización, hasta lo relacionado directamente con la actitud de los pobladores de la comunidad, quienes no reconocen la infestación por Ae. aegypti como un problema sanitario.

$C x$. quinquefasciatus se presentó como la segunda especie en orden de frecuencia y diversidad en los contenedores artificiales evaluados (Tablas 1 y 2, Figuras 1 y 2). Este mosquito es muy común en regiones tropicales y subtropicales y se ha asociado con los fenómenos de sustitución del hábitat en aquellos

Tabla 1. Frecuencia relativa de larvas de culícidos por criadero (Ii) observada durante la estación seca del 2003

\begin{tabular}{lcccccrr}
\hline Especie de culícido & Estañón & \multirow{2}{*}{ Balde } & Maceta & Florero & Lata & promedio & D \\
\hline Aedes aegypti & 0,400 & 0,180 & 0,220 & 0,090 & 0,040 & $0,180 *$ & 0,120 \\
Culex quinquefasciatus & 0,090 & 0,180 & - & - & 0,040 & 0,060 & 0,060 \\
\hline
\end{tabular}

$* \mathrm{p}<0,05$

Tabla 2. Frecuencia relativa de larvas de culícidos por criadero (Ii) observada durante la estación lluviosa de 2003

\begin{tabular}{|c|c|c|c|c|c|c|c|c|c|c|}
\hline \multicolumn{4}{|c|}{ Especie de culícido } & \multicolumn{3}{|c|}{ Contenedores } & \multirow[b]{2}{*}{ Bebedero } & \multicolumn{3}{|c|}{ Ii } \\
\hline & Estañón & Balde & Maceta & Florer & Llanta & Pila & & Lata & promedio & DS \\
\hline Ae. aegypti & 0,079 & 0,160 & 0,016 & 0,016 & 0,190 & 0,007 & 0,016 & 0,050 & $0,1000 *$ & 0,120 \\
\hline Cx. quinquefasciatus & is $\quad-$ & 0,040 & - & - & 0,023 & - & - & - & 0,0080 & 0,013 \\
\hline Cx. restuans & - & 0,007 & - & - & - & - & - & - & 0,0007 & 0,002 \\
\hline Cx. coronator & 0,007 & 0,007 & - & - & - & - & - & - & 0,0033 & 0,005 \\
\hline L. durhamii & - & 0,007 & - & - & - & - & - & 0,007 & 0,0015 & 0,003 \\
\hline
\end{tabular}

$* \mathrm{p}<0,05$ 
lugares donde se ha documentado una disminución o erradicación de Ae. aegypti luego de una campaña de intervención ${ }^{2}$.

Larvas de $C x$. restuans, $C x$. coronator y $L$. durhamii sólo fueron evidenciadas durante la estación lluviosa, con mucho menor frecuencia que Ae. aegypti y $C x$. quinquefasciatus. Las larvas de estos mosquitos se suelen encontrar en gran variedad de depósitos de agua natural y contenedores artificiales, por lo que su hallazgo en estos últimos no resulta extraño en comunidades urbanas y periurbanas ${ }^{11,12} . C x$. restuans y $C x$. coronator son especies propias del Continente Americano y tienen la característica de que sus adultos, a pesar de que son marcadamente ornitofílicos, también pueden alimentarse de sangre de mamíferos ${ }^{11}$. Por esta razón se podrían desempeñar como transmisores de virus aviares al ser humano y animales domésticos. En este sentido, es importante mencionar que el Virus del Nilo Occidental (WNV), capaz de producir cuadros de encefalitis en humanos y cuyos reservorios principales son ciertas especies de aves, utiliza como posibles vectores a mosquitos con estas características ${ }^{4,13}$ ${ }^{-15}$. En condiciones experimentales $C x$. restuans, se desempeña como un vector eficiente para este virus $^{16}$ y en zonas endémicas de Norteamérica, ha sido posible el asilamiento viral del WNV a partir de este mosquito ${ }^{13}$. Por lo tanto, esta especie podría desempeñar un papel muy importante en el mantenimiento de los ciclos enzóoticos virales ${ }^{15}$.

$C x$. coronator es una especie común en el continente, y también ha sido relacionado con la transmisión de ciertos virus como el virus de la encefalitis equina de San Luis (SLE) y el virus de la encefalitis equina de Venezuela (VEE) ${ }^{3}$. Considerando la expansión continental de la virosis por WNV, $C x$. coronator se podría perfilar como una de las posibles especies vectoras para esta región del continente.

La ocurrencia de criaderos mixtos (Figuras 1 y 2) permite suponer que estas especies son altamente tolerantes, por lo que la competencia por el nicho ecológico se ve minimizada y se incrementan las probabilidades de éxito en la colonización de nuevos ambientes.

La identificación y conciencia acerca de la presencia de estas especies de mosquitos en los ambientes urbanos y periurbanos adquiere cada vez más importancia de cara a poder perfeccionar las campañas de prevención y control por dengue y a la vez hacer prospección de posibles enfermedades emergentes que utilicen a estos mosquitos como sus eventuales vectores.

\section{RESUMEN}

Se realizó una encuesta larval por mosquitos urbanos en contenedores artificiales intra y peridomiciliares en la comunidad "La Carpio", San José, Costa Rica. "La Carpio" es una comunidad que sufrió un brote de dengue en los últimos meses de la estación lluviosa de 2002. En 2003, fueron visitadas 369 viviendas durante la estación seca y 582 en la lluviosa, para identificar los potenciales sitios de multiplicación de mosquitos. 1.160 contenedores fueron identificados y 152 de ellos $(13,0 \%)$ estuvieron positivos por larvas de al menos una especie de mosquitos. La presencia de larvas de dos especies de mosquitos en un mismo contenedor fue observada en 9 contenedores $(0,78 \%)$. Con el fin de estimar la diversidad larval de mosquitos, se calculó un índice de infestación por especie para cada tipo de contenedor. Cinco especies de larvas de mosquito fueron identificadas: Aedes aegypti, Culex quinquefasciatus, $C x$. restuans, $C x$. coronator y Limatus durhamii. Ae. aegypti fue la especie que se encontró con mayor frecuencia y fue la que mostró los índices de diversidad más altos para los contenedores identificados ( $\mathrm{p}<$ 0,05). La presencia de otras especies de dípteros diferentes a Ae. aegypti podría enmascarar el éxito en las campañas preventivas por dengue. Además, estos mosquitos podrían facilitar la transmisión de otras enfermedades emergentes como la encefalitis por el Virus de Nilo Occidental.

\section{REFERENCIAS}

1.- BISSET J, MARQUETTI M. Comportamiento relativo de las densidades larvales de Aedes (S) aegypti y Culex (C) quinquefasciatus durante la etapa intensiva de la campaña anti-A. aegypti. Rev Cub Med Trop 1983; 35: 176-180.

2.- BISSET J, MARQUETTI M, GONZÁLEZ B et al. La abundancia larval de mosquitos urbanos durante la campaña de erradicación del Aedes aegypti (Linnaeus, 1762) y del dengue en Cuba (1981-82). Rev Cub Med Trop 1985; 37: 161-8.

3.- ALMIRON W. Culicidae (Diptera) de la provincia de Córdoba. En: Salomón, O. Actualizaciones en artropodología sanitaria argentina. Fundación Mundo Sano, Buenos Aires, Argentina, 2002. 302 pp.

4.- PETERSEN L R, HUGHES J M. West Nile Virus encephalitis. N Engl J Med 2002; 347: 1225-6. 
5.- ORGANIZACIÓN PANAMERICANA DE LA SALUD. Tamaño de la muestra en encuestas larvales de Aedes. En: Dengue y dengue hemorrágico en las Américas. Organización Panamericana de la Salud, Publicación científica 548, Washington, D C 1997. 109 pp.

6.- VARGAS M. Clave de para los géneros de larvas de mosquitos (Diptera: Culicidae) de Costa Rica. En: El mosquito, un enemigo peligroso (Diptera: Culicidae). Editorial Universidad de Costa Rica, San José, Costa Rica, 1998. 264 pp.

7.- CENTERS FOR DISEASE CONTROL AND PREVENTION. Vector topics $\mathrm{N}^{\circ} 4$. Biology and control of Ae. aegypti. U. S. Department of Health, Education, and Welfare, Atlanta, GA, 1979. $68 \mathrm{pp}$.

8.- GONZÁLEZ R, DARSIE RF. Clave ilustrada para la determinación genérica de larvas de Culicidae de Colombia y el Nuevo Mundo. Bol Mus Ent Univ Valle 1996; 4: 21-37.

9.- DYAR HG. The mosquitoes of the Americas. Carnegie Institute. Publication N³87, Washington D C, 1928. $616 \mathrm{pp}$.

10.- DANIEL W. Pruebas de hipótesis. En: Bioestadística: Base para el análisis de las ciencias de la salud, Editorial Limusa, México, 1988. 667 pp.

11.- MARTINS DA SILVA A. Imaturos de mosquitos (Diptera, Culicidae) de áreas urbana e rural no norte do estado do Paraná, Brasil. Iheringia Ser Zool Porto Alegre 2002; 92: 31-6.

12.- MEANS R. Mosquitoes of New York. Part II. Genera of Culicidae other than Aedes ocurring in New York. Bulletin 430b, New York State Museum, Albany, 1987. $180 \mathrm{pp}$.
13.- APPERSON C S, HARRISON B A, UNNASCH T H, et al. Host.feeding habits of Culex and other mosquitoes (Diptera: Culicidae) in the Borough of Queens in New York City, with characters and techniques for identification of Culex mosquitoes. J Med Entomol 2002; 39: 777-85.

14.- SARDELIS M R, TURELL M J, DOHM D J, O'GUINN M L. Vector competencie of selected norh american Culex and Coquillettidia mosquitoes for West Nile Virus. Emer Infect Dis 2001; 7: 1018-22.

15.- ANDREADIS T G, ANDERSON J F, VOSSBRINCK C R. Mosquito surveillance for West Nile Virus in Connectitcut, 2000: Isolation from Culex pipiens, $C x$. restuans, $C x$. salinarius, and Culiseta melanura. Emer Infect Dis 2001; 4: 670-4.

16.- KULASEKERA V, KRAMER L, NASCI R et al. West Nile Virus infection in mosquitoes, birds, horses, and humans, Staten Island, New York, 2000. Emer Infect Dis 2001; 7: 722-5.

Agradecimientos: Los autores quieren expresar su agradecimiento al Dr. Oscar Montero, a la Lic. Fredda Wilhem y al personal asistencial del EBAIS de la comunidad "La Carpio" por la colaboración prestada; al Dr. Mario Vargas Vargas del Departamento de Parasitología de la Facultad de Microbiología en la Universidad de Costa Rica (UCR) por el material bibliográfico suministrado. También a los estudiantes que participaron en el Trabajo Comunal Universitario TC-492 y a la Sección de Trabajo Comunal Universitario de la Vicerrectoría de Acción Social (UCR) por su apoyo económico y logístico a este trabajo.

\section{ATENCION A LOS AUTORES}

PARA AGILIZAR LA EDICION DE PARASITOLOGIA LATINOAMERICANA, LOS AUTORES DEBERAN ENVIAR POR CORREO LOS ARTICULOS CIENTIFICOS GRABADOS EN UN DISKETTE DE $31 / 2$ EN WORD 6.0 O SUPERIOR JUNTO CON EL MANUSCRITO DEL TRABAJO A PUBLICAR O ADJUNTARLO POR CORREO ELECTRONICO A halcaino@uchile.cl 